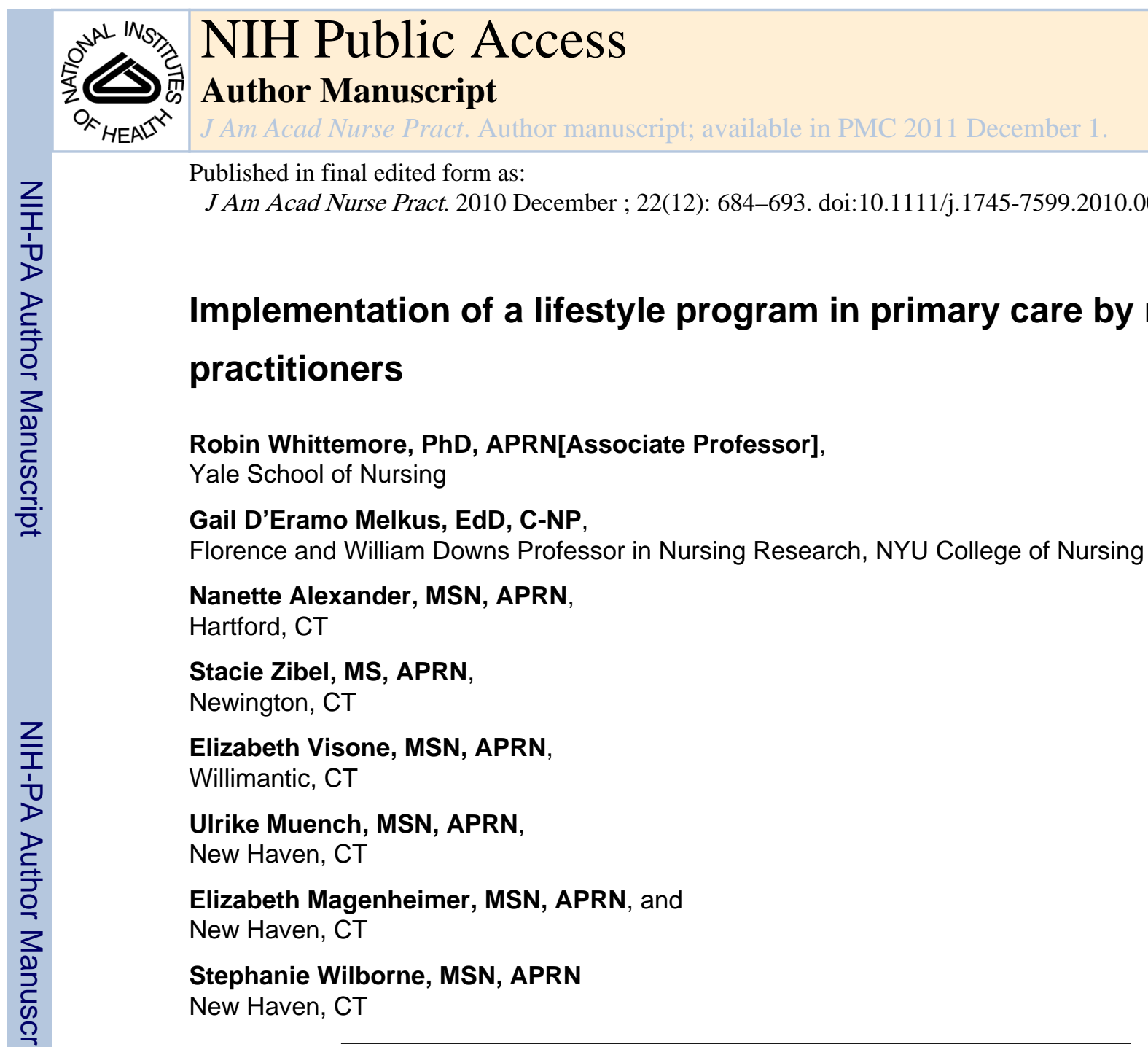

Type 2 diabetes (T2D) is emerging as a public health epidemic with approximately 24 million persons in the U.S. affected (National Institute of Diabetes and Digestive and Kidney Diseases [NIDDK], 2008). Racial and ethnic minorities, such as African Americans and Hispanics, have an increased risk as they are twice as likely as non-Hispanic whites of similar age to develop T2D. T2D is the primary cause of blindness, renal failure, and nontraumatic amputation in adults in the U.S. T2D also increases the risk of heart disease and stroke 2- to 4-fold (Centers for Disease Control \& Prevention [CDC], 2008]). Complications often occur concomitantly and contribute to disability, personal suffering, and considerable societal costs. In the U.S., the economic costs of diabetes in 2007 were estimated to be $\$ 172$ billion, an increase of 32\% from 2002 American Diabetes Association (ADA, 2003,2008). Therefore, the greatest opportunity to address the individual and societal costs of T2D is through disease prevention.

T2D is a heterogenous disease characterized by hyperglycemia due to dysfunction fo pancreatic islet-cells, increased hepatic glucose production, and insulin resistance (IR). T2D is preceded by a sustained period of impaired glucose tolerance (IGT) or less severe disturbances in glucose metabolism (Haffner, 2000). A diagnosis of IGT contributes to a high likelihood of developing T2D and a 1.5-fold risk of cardiovascular disease compared to those with normal blood glucose levels (Coutinho, Gerstein, Wang \& Yusuf, 1995; DECODE Study Group, 2001). Approximately 57 million adults in the U.S. have IGT

Corresponding Author: Robin Whittemore, Yale School of Nursing, 100 Church Street South, New Haven, CT 06536-0740, robin.whittemore@yale.edu. 
(CDC, 2008). IGT often occurs in conjunction with a constellation of cardiovascular risk factors including hypertriglyceridemia, low HDL cholesterol, and hypertension, which collectively comprise metabolic syndrome. Metabolic syndrome strongly predicts the development of T2D and cardiovascular disease (Ley et al., 2009; Lorenzo et al., 2003). A recent national survey (National Health and Nutrition Examination Survey [NHANES] 2003-2006) indicates a 34\% prevalence of metabolic syndrome in U.S. adults (Ervin, 2009).

While genetic factors contribute to the development of IGT, metabolic syndrome, and T2D, there is strong evidence that the risk factors of obesity and physical inactivity are the principal nongenetic determinants of these conditions (Ford, Giles, \& Mokdad, 2004; Krista et al., 2003). The increased prevalence of IGT, metabolic syndrome and T2D has coincided with an alarming increase in obesity in the U.S. Current estimates are that approximately 97 million adults in the U.S. are overweight or obese and thus at risk for the development of IGT, metabolic syndrome, and T2D (CDC, 2008).

Risk for T2D can be determined and T2D can be delayed or prevented through lifestyle programs. International clinical trials have demonstrated a $31-58 \%$ reduction in T2D for adults with IGT who participated in lifestyle programs compared to a control group (Tuomilehto et al., 2001; Yamoka \& Tango, 2005), sustained up to 7 years (Lindstrom et al., 2006). The Diabetes Prevention Program (DPP), a large multicenter clinical trial in the U.S. with a diverse sample of adults, demonstrated a significant decrease in the progression from IGT to T2D for those who participated in a lifestyle program that promoted healthy eating and physical activity (Knowler et al., 2002;). A significant association was demonstrated between T2D prevention and the degree to which participants were able to make recommended lifestyle changes (Tuomilehto et al., 2001). Lifestyle changes of healthy eating and physical activity contribute to weight loss and improvement in insulin resistance, which have been shown to predict progression to T2D in at-risk adults (Kitabchi et al., 2005). It is important to mention that the lifestyle goals that contributed to T2D prevention in these studies were modest. Lifestyle goals included weight loss of 5-7\% of body weight, reduction in fat intake to $<30 \%$, reduction in saturated fat intake to $<10 \%$, an increase in fiber to $15 \mathrm{gm} / 1000 \mathrm{kcal}$, and exercise (30 minutes $5-7$ days per week). These lifestyle changes also reduced cardiac risk factors of participants (i.e., hypertension, hyperlipidemia) (Tuomilehto et al., 2001).

Several trials using pharmacological agents have also demonstrated a significant decrease in the risk of developing T2D by 31-56\% in at-risk populations (Chiasson et al., 1998;

Knowler et al., 2002). The recent DPP clinical trial compared the efficacy of a pharmacologic agent (metformin) with a lifestyle program; the lifestyle program had nearly twice the effectiveness in preventing T2D compared to the pharmacologic agent (Knowler et al., 2002). The greater comparative benefit of lifestyle change has resulted in recommendations by the ADA advocating for lifestyle change as the first line of treatment to prevent or delay T2D (ADA, 2009). Results of a cost analysis of the DPP indicate a favorable cost-effective profile of the lifestyle program at any adult age (Herman et al., 2005). A considerable challenge is how to provide evidence-based lifestyle programs, such as the DPP, to populations at-risk.

\section{Diabetes Prevention Program}

A patient-centered multi-faceted approach, providing educational, behavioral, and psychosocial strategies, was the underlying premise of the DPP to promote lifestyle change in adults at-risk for T2D. The DPP was based on behavioral science and included the following components: a collaborative model of care, education, behavioral support (ie., goal setting, problem-solving), and motivational interviewing (DPP Research Group, 1999). 
Research supports that education and behavioral support are effective in changing health behaviors (Foster, Makris \& Bailer, 2005; Nothwehr \& Yang, 2007). Motivational interviewing (MI) is a counseling method used to enhance motivation to change by exploring and addressing ambivalence to change. When implementing MI, providers encourage individuals to set the agenda, discuss challenges to behavior change, and selfselect behavior change goals (Rollnick, Miller \& Butler, 2007). The effectiveness of motivational interviewing has been demonstrated in a wide range of interventions, including interventions to promote nutrition and physical activity (Resnicow et al., 2002; West et al., 2007).

Goals of the DPP were for all participants to lose $7 \%$ of their initial body weight within 6 months and to participate in at least 150 minutes of moderate physical activity (e.g., brisk walking) per week. Dietary modification to reduce fat and caloric intake was also part of the program, yet was not an explicit goal. Weight loss of 5-7\% in adults at risk for T2D decreases insulin resistance and has been shown to reduce the risk for T2D (Colman et al., 1995; Rana, Li, Manson \& Hu, 2007). In the DPP, modest weight loss was a predictor of risk reduction, with a $16 \%$ reduction in diabetes risk per kilogram of weight loss (Hamman et al., 2006). Exercise reduces risk factors for IGT and T2D by decreasing glucosestimulated insulin production, increasing insulin sensitivity, and decreasing abdominal adiposity (Bloem \& Chang, 2008; Pratley et al., 2000). Dietary modification can improve lipid profiles and is an important component to weight loss and the improvement of T2D risk factors.

In order to promote and facilitate weight loss, the DPP consisted of a core curriculum (6 months) and a maintenance program (up to 3 years). The core curriculum comprised 16 individualized sessions with the case manager (45 minutes) that included a private weigh-in, review of self-monitoring records, education on a specified topic, ongoing identification of barriers to weight loss and exercise, and the development of action plans. The maintenance program was less structured than the core curriculum and consisted of group and individual sessions provided by case managers addressing ongoing exercise, weight loss, and behavioral issues. In addition, motivational campaigns and incentives were provided (DPP Research Group, 1999).

\section{Translation of Diabetes Prevention Program}

Approaches to translate T2D prevention programs into different settings have been investigated. A systematic review of interventions to prevent or delay T2D (n=9) reported variable treatment models and modest improvements in outcomes (Satterfield et al., 2003). The majority of studies in this review used one-group designs, were not based on the DPP curriculum, and few measured plasma glucose or insulin resistance More recently, lifestyle programs translating the DPP to the community, workplace, or churches have demonstrated significant weight loss of participants and improvements in IR and lipid profiles in onegroup designs (Aldana et al., 2006; Amundsun et al., 2009; Boltri et al., 2008; Laatikainen et al., 2007; Seidel et al., 2008). Attrition in these studies varied from 8-50\%, with less attrition up to 1 year when interventions were provided in familiar places. A pilot randomized trial translating a group-based DPP to the YMCA setting has demonstrated preliminary efficacy in weight loss and improving total cholesterol (Ackermann, Marrero, Zhou \& Brizendine, 2007). Our research team conducted a pilot randomized trial, modifying the DPP for implementation in the primary care setting by nurse practitioners (NPs) and comparing the preliminary efficacy to an enhanced standard care group. Lifestyle participants demonstrated a trend toward greater weight loss $(\mathrm{p}=.08)$ and improved exercise behavior ( $\mathrm{p}=.08$ ). Twenty-five percent of participants met weight loss goals in the lifestyle program compared to $11 \%$ in standard care (Whittemore et al., 2009). The uniqueness of our 
approach was aligning the professional values and qualifications of NPs in primary care with the purpose and needs of the program (health education, counseling, screening, treatment, and follow-up). While results of this study have been reported, the aim of this analysis is to describe the implementation process and participant satisfaction with the lifestyle program compared to enhanced standard care. Recommendations for practice will be discussed.

\section{Method}

\section{Design}

A mixed-method clinical trial design was used to complete the study aims of the primary study and this secondary analysis (Whittemore et al., 2009). Institutional Review Board approval was obtained from all sites affiliated with the study.

\section{Sample}

A convenience sample of 4 NP sites was recruited from a regional practice-based research network in New England. A cluster randomization procedure randomly assigned sites into 2 groups: a treatment group (who implemented the lifestyle change program) and a control group (who provided the enhanced standard care control condition). One NP randomized to the treatment group had difficulty with recruitment due to practice patterns of prescribing metformin for adults at-risk for T2D (study exclusion criteria). An additional NP site was recruited to supplement this site. NP's recruited a convenience sample ofadults at-risk for T2D ( $n=58)$ ( 28 treatment and 30 control group participants). The sample size was determined by a power analysis, recruiting approximately $20 \%$ of what would be necessary for a full test of the hypothesis.

\section{Procedures}

Enhanced Standard Care-Upon entrance into the study, all participants (regardless of group assignment) received written information, a 20-30 minute individual session with their NP on lifestyle change and T2D prevention, and a 45-minute individual session with a nutritionist hired for the study. This enhanced standard care approach represents current treatment recommendation for T2D prevention (ADA, 2004). Specifically, participants were counseled to lose $5-10 \%$ of their body weight through lifestyle change (healthy eating andexercise). Exercise recommendations were for participants to gradually work toward 30 minutes of exercise (ie., walking) 5 days per week.

Lifestyle Change Program-The lifestyle change program was based on the protocol for the DPP. The goals for this program were identical to the enhanced standard care condition; however, the intervention included more sessions and content on the challenges of lifestyle change (DPP Research Group, 1999). The lifestyle change program provided: a) education on nutrition, exercise, and type 2 diabetes prevention that included culturally relevant handouts (ie., recipes); b) behavioral support of identifying lifestyle change strategies and problem-solving barriers to change; and c) motivational interviewing. Details of the lifestyle program have been previously reported (Whittemore et al., 2009) and consisted of 6 inperson sessions and 5 phone sessions delivered over approximately 6 months.

All NPs received a 2-hour training session on study procedures, the protocol for the enhanced standard care session, and documentation forms. NPs of the lifestyle program received additional training on the protocol for the lifestyle sessions and MI. This training consisted of self-study (selected reading and a 45-minute video on MI) and two 2-hour workshops provided by a clinical psychologist with expertise in MI. One workshop was provided at the beginning of the study and the other approximately 4 months later. The primary investigator met with all NPs every several months to discuss study implementation. 
NPs of the enhanced standard care condition received MI training at the completion of the study.

NPs and nutritionists were compensated modestly for study training time. NPs or NP practices and nutritionists were also reimbursed for sessions provided to study participants. NP practices and participants did not incur any expenses related to the study nor were health insurances billed for services provided.

\section{Outcome Measures}

Implementation-Data were collected on attendance, attrition, and intervention fidelity. Intervention fidelity data consisted of NP documentation of each session. Documentation forms were developed that included a check-list of all of the components of a particular session and a column for NPs to record pertinent information related to protocol implementation. Protocol implementation was calculated by dividing the number of items of the protocol by the number of items completed per session. NPs also completed a Lifestyle Counseling Questionnaire prior to beginning the study (Simkin-Silverman \& Wing, 1997). This questionnaire asks 4 items that ask how prepared and effective providers feel they are in providing nutrition, weight, and exercise advice. Each item is constructed with a 5-point Likert scale. NPs were interviewed at baseline to identify current practice patterns on diabetes prevention. Lastly, NPs were interviewed at 3 and 6 months to identify the successes and challenges of implementation.

Satisfaction-Participant satisfaction was evaluated with a Program Satisfaction Survey. The satisfaction survey was a 7-item scale modified from the Diabetes Treatment Satisfaction Survey (Bradley, 1994) to evaluate a diabetes prevention program. Internal consistency of the original scale (alpha $=.82)$ and the modified scale in this study (alpha $=.86$ ) were adequate. Participants were also interviewed regarding their satisfaction with the program $(n=24)$.

\section{Data Analysis}

Quantitative data were entered into databases (Microsoft Access or Excel) using an automated Teleform system. Descriptive statistics were calculated using frequency distributions and summary statistics (SAS). Qualitative data were obtained from transcribed interviews and written notes which were analyzed using content analysis techniques (Miles \& Huberman, 1994). The following steps were accomplished: a) transcribing the taped interviews; b) checking transcripts against tapes for accuracy; c) developing coding categories; d) assigning codes of text with appropriate checks; and d) reviewing coded data to identify themes across participants.

\section{Results}

Sample

Seven NPs from 5 study sites participated in the study (2 enhanced standard care sites and 3 lifestyle program sites). Health concerns required one NP to drop out of the study and one to take a 6-week hiatus from the study. An NP replacement was hired for the NP who could no longer participate in the study. Despite these challenges, study protocols were successfully implemented and participants were retained.

Fifty-eight patient participants were enrolled in the study. The response rate to in-person recruitment at NP practices was $70 \%$ and attrition rate at 6 months was $12 \%$. Participants who did not complete the study were younger, had higher BMI, and lower low-density lipoproteins ( $\mathrm{p}<.05$ ). Reasons for attrition included: personal reasons such as medical 
problems $(n=4)$, lost to follow-up $(n=2)$, or preference for alternate treatment $(n=1)$. The sample represented diverse (45\% White; $34 \%$ Black; $21 \%$ Hispanic), primarily female (92\%), obese, moderately-low income adults at risk for T2D. Elevated depressive symptoms were reported by $33 \%$ of the sample. Other sample characteristics have been previously reported (Whittemore et al., 2009).

\section{Implementation}

The Lifestyle Counseling Questionnaire was completed by all NPs prior to study implementation (Table 1). NPs reported that they felt well-prepared and moderately effective in providing lifestyle counseling to adults at-risk for T2D in their practice settings. However, none of the NPs had a structured approach to T2D prevention in their practice. NPs reported that lifestyle counseling often occurred informally within the context of primary care appointments.

Participant attendance for in-person sessions was high at $96 \%$ across all sessions and all participants. Reminder phone calls for appointments and flexible rescheduling enhanced attendance. NP phone call completion for the lifestyle program was only $37 \%$ due to scheduling difficulties (both providers and participants). NPs would schedule phone appointments and make 1-2 additional attempts if unable to reach the participant at the designated time. The protocol specified that NP sessions were to be approximately 20 minutes and the nutritionist appointment 45 minutes. The average time of the enhanced standard care NP session that all study participants received was 26 minutes and the average time of nutrition sessions was 58 minutes. With respect to the lifestyle program sessions, the average time of sessions was 27 minutes. NPs reported that it was challenging to complete all aspects of the study protocol with a 20 minute session, particularly when participants wanted more information or had psychosocial issues. Participants of the enhanced standard care group received approximately 1.5 hours of professional support and participants of the lifestyle group received approximately 4.1 hours of professional support compared to 12-16 hours of individual sessions in the DPP core curriculum. Implementation of the lifestyle program took approximately 2 months longer than anticipated due to NP illnesses, rescheduling of participant appointments, and the end of the year holidays.

With respect to implementing the study protocols, in-person sessions were implemented with success. NP Implementation of the standard care protocol was 80\%, implementation of the standard care nutrition protocol was $92 \%$, and NP implementation of the lifestyle protocol was $76 \%$ across all sessions. Overall protocol implementation did not decline over time in the lifestyle group (Figure 1). NPs of the lifestyle group reported that the program implementation was easier over time and that they were able to individualize the session content to best meet the needs of a participant. For example, if more time was required to discuss barriers to exercise or psychosocial issues, new educational content was reviewed briefly and participants were advised to read the material more thoroughly at home. NPs reported that the most challenging aspect of implementing the lifestyle program was with MI, a new behavioral strategy for all NPs. NPs reported difficulty in helping participants build motivation to change and align their behaviors with personal values and goals.

\section{Participant Satisfaction}

Participants of the lifestyle program were more satisfied with the program $(\mathrm{p}=.05)$.

Differences with satisfaction were also expressed by participants who were interviewed at the end of program $(n=26)$. Lifestyle participants reported that they enjoyed the study and felt that it was a good experience. They felt that the number and length of sessions were adequate and they appreciated the flexibility in scheduling appointments. Participants of the control group reported that the number of appointments was not enough and that they 
needed more sessions and follow-up on progress. For example, one participant stated that her motivation declined after she realized no one was tracking her.

Participants were asked what motivated them to make lifestyle changes and complete the program. Some participants were concerned about being at risk for T2D, not wanting to have to take medicine, and wanting to know more about health. Others wanted to lose weight, were motivated by having to be weighed on a regular basis, and felt that having to be accountable to someone for their behavior was important. Formal meetings and feeling like they completed the program were also reported as motivating to some participants.

Participants of both groups commented very positively on having the program provided in a familiar place by a familiar provider. As one female participant stated, "Knowing my NP made me more comfortable...the rapport that we have was helpful... and it motivated me to come to sessions."

When asked what they learned in the program, the most common responses were that participants learned about portion sizes, food labels, better food choices, the relationship of weight loss to prevention of diabetes, and the importance of exercise. Several participants of the lifestyle group reported that they learned about themselves. "The program made me more aware of my bad habits." "I learned about the excuses I make not to exercise." Others learned how to encourage themselves to do things they don't enjoy but that are healthy, such as exercise. One participant learned "to continue even after messing up."

Behavior changes reported by participants included positive dietary changes such as increasing vegetables, decreasing portions, eliminating cold cuts, sugar, and soda, buying less junk food, reading labels, and eliminating snacking at night. Participants also reported exercising, making exercise a priority, and increasing physical activity during the day. Some participants were looking into other weight control or exercise programs at the completion of the study. Others were using the internet more to get information and recipes.

Barriers to dietary change included stress, time, and financial resources. Some participants reported that eating a healthy diet was expensive and time-consuming. Others had the greatest difficulty with limiting favorite cultural foods (ie. rice and beans), portion control, or social engagements. Barriers to exercise included the weather and other physical conditions that caused pain or limited mobility.

Suggestions for improvement included more sessions and content by control group participants, greater support for exercise, and more sessions with the nutritionist. Many participants recognized the challenges of persisting with lifestyle change and the need for ongoing professional support.

\section{Discussion}

Successful implementation of study protocols and participant satisfaction with the lifestyle program are important results of this study. Attendance for program sessions was high and attrition was low, critical elements to improving T2D prevention in diverse populations. Previous research of behavioral or psychological interventions in primary care has demonstrated high attrition (30-53\%) and issues in implementation (Graffagnino et al., 2006; Zayas, McKee \& Jankowski, 2004). Participants were particularly satisfied with having a lifestyle program implemented in their primary care setting by a familiar provider. NPs were able to implement the standard care and lifestyle program aimed at T2D prevention within the context of primary care (ie. 20 minute sessions) and without lengthy training. The finding that NPs were able to implement a lifestyle program for T2D is important, as primary care physicians have reported challenges to lifestyle counselling 
(Kristeller \& Hoerr, 1997; Larme \& Pugh, 1998). Factors that appear critical to enhancing implementation of a T2D prevention program in primary care include developing a structured program for T2D prevention, referring patients at risk for T2D to community programs, and learning about MI.

\section{Developing a Structured Program for T2D Prevention}

Standards of care for diabetes prevention are available and include assessing risk, promoting lifestyle change, providing follow-up, and treating cardiovascular risk factors such as dyslipidemia, hypertension, and tobacco use (ADA, 2004; 2009;Indian Health Services, 2006). These components can be modified for primary care settings to meet the unique needs of a clinic or population served. While all NPs in this study reported that T2D prevention was important and assessed patients for risk, none had a standardized approach to lifestyle change and T2D prevention in their practice. Participants of this study, commented favorably on participating in a formal program and having to be accountable to their NP for their behaviors. Participants also indicated their need for regular follow-up over time.

NPs in this study expressed that calculating a 5-7\% weight loss goals for patients was something that they had not been doing with patients at risk for T2D but was a very effective way to introduce a T2D prevention program to patients. NPs also felt that having standardized handouts and chart forms facilitated their ability to provide T2D prevention counseling in a busy primary care practice. Modeled after this study, each session could include time for evaluating progress and problems, implementing appropriate behavioral counseling, and providing culturally relevant education. An example of a chart form is provided in Table 2. Behavioral strategies and possible education modules are identified in Table 3. Many organizations provide culturally relevant educational materials that could be incorporated into each education module (ie. National Diabetes Education Program).

Long-term follow-up to reinforce lifestyle goals, promote weight loss, and prevent T2D is recommended, however, the timing of follow-up has not been determined (ADA, 2004). Research supports the benefit of regular professional support in order to enhance lifestyle change and weight loss (Avenell et al., 2004). Frequent visits initially, followed by monthly visits, may be reasonable and would allow for ongoing education and behavioral support as well as the opportunity to follow-up for other comorbidity such as hypertension and dyslipidemia. While phone sessions were difficult for NPs in this study, they may be feasible in some settings. Email may also be an efficient mode of communication for lifestyle change follow-up. The actual timing of follow-up will need to be individualized based on patient, practitioner, and system characteristics (ie. reimbursement). Developing office systems to support ongoing follow-up, such as appointment reminders and rescheduling, appear critical to assure patient attendance.

\section{Referring to Community Resources}

NPs and participants highly valued the in-person session with a nutritionist. Most NPs felt that in-depth nutritional counseling was beyond their scope of practice and participants felt that the session with a nutritionist was very informative, helping to identify aspects of their diet that they could begin to change. Medical nutrition therapy for T2D prevention is often reimbursed by Medicare and $3^{\text {rd }}$ party payers, and should be considered as part of a T2D prevention program provided in primary care. Referral to other community resources may also be considered. For example, many diabetes education programs are beginning to provide T2D prevention classes which could provide additional educational and behavioral support for patients initiating lifestyle change. 


\section{Learning about MI}

NPs in this study reported confidence in providing education related to lifestyle change to prevent T2D. In addition, NPs were confident in their ability to collaboratively determine goals, assess progress and problems, and problem-solve barriers to lifestyle change. MI was a behavioral strategy that most NPs reported less confidence in their ability to implement. Because MI was an important component of the DPP, NPs who are interested in implementing a diabetes prevention program in their practice and do not have expertise in MI, would benefit from additional training. Numerous resources and workshops are available (Rollnick et al., 2007; Wagner \& Conners, 2009).

\section{Additional considerations}

While this study reports on the implementation of a lifestyle program to prevent T2D, focusing on lifestyle change, close attention should also be given to other cardiovascular risk factors, such as hypertension, dyslipidemia, metabolic syndrome, and tobacco use in adults at risk for T2D (ADA, 2009). In addition, screening for depression in adults at risk for T2D is indicated. Adults with prediabetes have a high prevalence of depression (Rubin et al., 2005) and depression may impede lifestyle change efforts (Lin et al., 2004). Treatment of depression in adults at risk for T2D may also improve insulin sensitivity (Wagner et al., 2009: Weber-Hamann et al., 2008). Lastly, metformin may be considered for those individuals who are at very high risk for developing T2D, although drug therapy is not recommended as a routine approach to prevent T2D unless other comorbidities such as hypertension and hyperlipidemia are present (ADA, 2009).

\section{Conclusion}

NPs have the expertise to manage the screening, treatment, and follow-up for the prevention of T2D in at-risk adults. Implementing a program to prevent T2D in primary care is feasible and acceptable to NPs and participants. Developing a structured program for assessing risk, promoting lifestyle change, and managing comorbidity for adults at-risk for T2D may improve outcomes. Developing a system that includes chart documents, educational handouts, and a schedule for follow-up is recommended. NPs may also want to consider including other health professionals and providing a team-based approach to diabetes prevention.

\section{Acknowledgments}

NIH/NIDDK R34DK070594; NIH/NCRR/CTSA Program Grant \# UL1 RR024139.

Alison Beale, RD; Diane Bussolini, RD; Jo Cecille Demarest, MS; Amy Triche, BA, Monika Haugstetter, BS, Felicia Lucas, BSN, Alyssa Roman, BA, Leah Swalley, BS, Tony Ma, PhD, Julie Wagner, PhD; Judith WylieRosett, EdD, RD.

\section{References}

Ackermann RT, Marrero DG, Zhou H, Brizendine EJ. Translating the diabetes prevention program into the community: The YMCA model. Diabetes. 2007;56(suppl):OR-0164.

Aldana S, Barlow M, Smith R, Yanowitz F, Adams T, et al. A worksite diabetes prevention program: Two-year impact on employee health. American Association of Occupational Health Nurses. 2006;54:389-95.

American Diabetes Association. Economic costs of diabetes in the US in 2002. Diabetes Care. 2003;26:917-32. [PubMed: 12610059]

American Diabetes Association. Economic costs of diabetes in US in 2007. Diabetes Care. 2008;31:120. 
American Diabetes Association. Standards of care in diabetes - 2009. Diabetes Care. 2009;32:S1. [PubMed: 19118285]

American Diabetes Association \& National Institute of Diabetes and Digestive and Kidney Diseases. Prevention or delay of type 2 diabetes. Diabetes Care. 2004;27 (Supple 1):S47-S54. [PubMed: 14693925]

Amundson HA, Butcher MK, Gohdes D, Hall TO, Harwell TS, Helgerson SD, Vanderwood KK. Translating the diabetes prevention program into practice in the general community. The Diabetes Educator. 2009;35:209-223. [PubMed: 19321807]

Avenell A, Broom J, Brown TJ, Poobalan A, Aucott L, et al. Systemic review of the long-term effects and economic consequences of treatments for obesity and implications for health improvement. Health Technology Assessment. 2004;8:1-182.

Bloem CJ, Chang AM. Short-term exercise improves \{beta -cell function and insulin resistance in older people with impaired glucose tolerance. Journal of Clinical Endocrinology \& Metabolism. 2008;93:387-92. [PubMed: 18000089]

Boltri JM, Davis-Smith YM, Seale JP, Shellenberger S, Okosun IS, Cornelius ME. Diabetes prevention in a faith-based setting: Results of translational research. Journal of Public Health Management Practice. 2008;14:29-32.

Bradley, C. Diabetes treatment satisfaction questionnaire. In: Bradley, C., editor. Handbook of psychology and diabetes. Australia: Harwook Academic Publishers; 1994. p. 111-132.

Centers for Disease Control and Prevention (CDC). National diabetes fact sheet: General information and national estimates on diabetes in the united states, 2007. Atlanta, GA: U.S. Department of Health and Human Services, Centers for Disease Control and Prevention; 2008.

Chiasson JL, Gomis R, Hanefeld M, Josse RG, Karaski A, Laakso M. The STOP-NIDDM trial: An international study on the efficacy of an alpha-glucosidase inhibitor to prevent type 2 diabetes in a population with impaired glucose tolerance: Rationale, design, and preliminary screening data: Study to prevent non-insulin-dependent diabetes mellitus. Diabetes Care. 1998;21:1720-5. [PubMed: 9773737]

Colman E, Katzel LI, Rogus E, Coon P, Muller D, Goldberg AP. Weight loss reduces abdominal fat and improves insulin action in middle-aged and older men with impaired glucose tolerance. Metabolism. 1995;44:1502-1508. [PubMed: 7476341]

Coutinho M, Gerstein HC, Wang Y, Yusuf S. The relationship between glucose and incident cardiovascular events: A metaregression analysis of published data from 20 studies of 95,783 individuals followed for 124 years. Diabetes Care. 1999;22:233-40. [PubMed: 10333939]

Decode Study Group. Glucose tolerance and cardiovascular mortality: Comparison of fasting and 2hour diagnostic criteria. Archives of Internal Medicine. 2001;161:397-405. [PubMed: 11176766]

DPP Research Group. Design and methods for a clinical trial in the prevention of type 2 diabetes. Diabetes Care. 1999;22:623-34. [PubMed: 10189543]

Ervin, RB. National Health Statistics Reports. Vol. 13. Hyattsville, MD: National Center for Health Statistics; 2009. Prevalence of metabolic syndrome among adults 20 years of age and over, by sex, age, race and ethnicity, and body mass index: United States, 2003-2006.

Ford ES, Giles WH, Mokdad AH. Increasing prevalence of the metabolic syndrome among U.S. adults. Diabetes Care. 2004;27:2444-2449. [PubMed: 15451914]

Foster GD, Makris AP, Bailer BA. Behavioral treatment of obesity. American. Journal of Clinical Nutrition. 2005;82(Suppl 1):230S-235S.

Graffagnino CL, Falko JM, La Londe M, Schaumburg J, Hyek MF, Shaffer LE, et al. Effect of a community-based weight management program on weight loss and cardiovascular disease risk factors. Obesity. 2006;14:280-288. [PubMed: 16571854]

Haffner SM. Obesity and the metabolic syndrome: The San Antonio Heart Study. British Journal of Nutrition. 2000;83:S67-70. [PubMed: 10889794]

Hamman RF, Wing RR, Edelstein SL, Lachin JM, Bray GA, Delahanty L, et al. Effect of weight loss with lifestyle intervention on risk of diabetes. Diabetes Care. 2006;9:2102-2107. [PubMed: 16936160] 
Herman WH, Hoerger TJ, Brandle M, Hicks K, Sorensen S, Zhang P, et al. The cost-effectiveness of lifestyle modification or metformin in preventing type 2 diabetes in adults with impaired glucose tolerance. Annals of Internal Medicine. 2005;142:323-332. [PubMed: 15738451]

Indian Health Service Division of Diabetes Treatment and Prevention. HIS guidelines for care of adults with prediabetes and/or the metabolic syndrome in clinical settings. 2006.

Kitabchi AE, Temprosa M, Knowler WC, Kahn SE, Fowler SE, Haffner SM, et al. Role of insulin secretion and sensitivity in the evolution of type 2 diabetes in the diabetes prevention program: Effects of lifestyle intervention and metformin. Diabetes. 2005;54:2404-2414. [PubMed: 16046308]

Knowler WC, Barrett-Connor E, Fowler SE, Hamman S, Lachin JM, Walker EA, et al. Reduction of the incidence of type 2 diabetes with lifestyle intervention or metformin. New England Journal of Medicine. 2002;346:393-403. [PubMed: 11832527]

Krista AM, Saremi A, Hanson RL, Bennett PH, Kokes S, Williams DE, Knowler WC. Physical activity, obesity, and the incidence of type 2 diabetes in a high-risk population. American Journal of Epidemiology. 2003;158:669-675. [PubMed: 14507603]

Kristeller JL, Hoerr RA. Physician attitudes toward managing obesity: Differences among six specialty groups. Preventive Medicine. 1997;26:542-549. [PubMed: 9245677]

Laatikainen, T.; Dunbar, JA.; Chapman, A.; Kilkkinen, A.; Vartiainen, E.; Heistaro, S., et al. Prevention of type 2 diabetes by lifestyle intervention in an australian primary health care setting: Greater green triangle (GGT) diabetes prevention project; BMC Public Health [Internet]. 2007. p. 3/2008Available from www.biomedicalcentral.com/1471-2458/7/249

Larme AC, Pugh JA. Attitudes of primary care providers toward diabetes: Barriers to guideline implementation. Diabetes Care. 1998;21:1391-1396. [PubMed: 9727882]

Ley SH, Harris SB, Mamkeesick M, Noon T, Fiddler E, Gittelsohn J, et al. Metabolic syndrome and its components as predictors of incident type 2 diabetes in an Aboriginal community. Canadian Medical Association Journal. 2009;17:617-624. [PubMed: 19289805]

Lin EHB, Katon W, Von Korff M, Rutter C, Simon G, et al. Relationship of depression and diabetes self-care, medication adherence, and preventive care. Diabetes Care. 2004;27:2154-2160. [PubMed: 15333477]

Lindstrom J, Ilanne-Parikka P, Peltonen M, Aunola S, Eriksson JG, Hemio K, et al. Sustained reduction in the incidence of type 2 diabetes by lifestyle intervention: Follow-up of the Finnish Diabetes Prevention Study. Lancet. 2006;368:1673-1679. [PubMed: 17098085]

Lorenzo C, Okoloise M, Williams K, Stern MP, Haffner SM. The metabolic syndrome as a predictor of type 2 diabetes: The San Antonio heart study. Diabetes Care. 2003;26:3153-3159. [PubMed: 14578254]

Miles, MB.; Huberman, AM. Qualitative data analysis. Sage Publication; Thousand Oaks: 1994.

National Institute of Diabetes and Digestive and Kidney Diseases. Fact Sheet: Type 2 Diabetes [Internet]. 2008. Available from: http://www.nih.gov/about/researchresultsforthepublic/Type2Diabetes.pdf

Nothwehr F, Yang J. Goal setting frequency and the use of behavioral strategies related to diet and physical activity. Health Education Research. 2007;22:532-538. [PubMed: 17032703]

Pratley RE, Hagberg JM, Dengel DR, Rogus EM, Muller DC, Goldberg AP. Aerobic exercise traininginduced reductions in abdominal fat and glucose-stimulated insulin responses in middle-aged and older men. Journal of American Geriatrics. 2000;48:1055-1061.

Rana JA, Li TY, Manson JE, Hu FB. Adiposity compared with physical inactivity and risk of type 2 diabetes in women. Diabetes Care. 2007;30:53-58. [PubMed: 17192333]

Resnicow K, DiIorio C, Soet J, Borrelli B, Hecht J, Ernst D. Motivational interviewing in health promotion: It sounds like something is changing. Health Psychology. 2002;21:444-451. [PubMed: 12211511]

Rollnick, S.; Miller, WR.; Butler, CC. Motivational interviewing in health care. New York: Guilford Press; 2007.

Rubin RR, Knowler WC, Ma Y, Marrero DG, Edelstein SL, Walker EA, et al. Depression symptoms and antidepressant medicine use in diabetes prevention program participants. Diabetes Care. 2005;28:830-837. [PubMed: 15793181] 
Satterfield DW, Volansky M, Caspersen CJ, Engelgau MM, Bowman BA, Gregg EW, et al. Community-based lifestyle interventions to prevent type 2 diabetes. Diabetes Care. 2003;26:26432652. [PubMed: 12941733]

Seidel MC, Powell RO, Zgibor JC, Siminerio LM, Piatt GA. Translating the diabetes prevention program into an urban medically underserved community: A non-randomized prospective intervention study. Diabetes Care. 2008;31:684-9. [PubMed: 18252904]

Simkin-Silverman LR, Wing RR. Management of obesity in primary care. Obesity Research. 1997;5:603-612. [PubMed: 9449146]

Tuomilehto J, Lindstrom J, Eriksson JG, Valle TT, Hamalainen H, Ilanne-Parikka P, et al. Prevention of type 2 diabetes mellitus by changes in lifestyle among subjects with impaired glucose tolerance. New England Journal of Medicine. 2001;344:1343-1350. [PubMed: 11333990]

Wagner, CC.; Conners, W. Motivational interviewing: Training. 2009 [Accessed March 8, 2009]. www.motivationalinterviewing.org/training/index.html

Wagner J, Allen N, Swalley L, Melkus G, Whittemore R. Depression, Depression Treatment, and Insulin Sensitivity in Adults at Risk for Type 2 Diabetes. Diabetes. 2009;58(Supp 1):A476.

Weber-Hamann B, Gilles M, Schilling C, Onken V, Frankhauser P, Kopf D, et al. Improved insulin sensitivity in 51 nondiabetic depressed patients remitting during antidepressive treatment with mirtazapine and venlafaxine. Journal of Clinical Psychopharmacology. 2008;28:581-584. [PubMed: 18794665]

West DS, DiLillo V, Bursac Z, Gore SA, Greene PG. Motivational interviewing improves weight loss in women with type 2 diabetes. Diabetes Care. 2007;30:1081-1088. [PubMed: 17337504]

Whittemore R, Melkus G, Wagner J, Northrup V, Dzuria J, Grey M. Translating the Diabetes Prevention Program to primary care: A pilot study. Nursing Research. 2009;58:2-12. [PubMed: 19092550]

Yamaoka K, Tanto T. Efficacy of lifestyle education to prevent type 2 diabetes: A meta-analysis of randomized controlled trials. Diabetes Care. 2005;28:2780-2786. [PubMed: 16249558]

Zayas LH, McKee MC, Jankowski KR. Adapting psychosocial intervention research to urban primary care environments: A case example. Annals of Family Medicine. 2004;5:504-508. [PubMed: 15506589] 


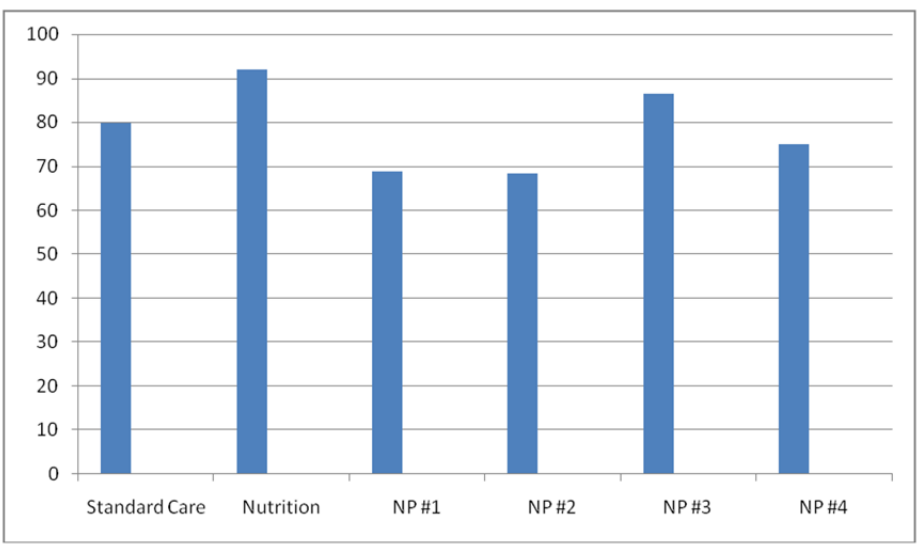

Figure 1.

Percent of Protocol Implementation by Session 
Table 1

Results of Lifestyle Counseling Questionnaire

\begin{tabular}{|l|l|}
\hline Item & Mean (SD) \\
\hline How well prepared to provide weight and exercise advise (range 0-5) & $4.5(.55)$ \\
\hline How effective in providing weight and exercise advise (range 0-5) & $3.75(.42)$ \\
\hline How often counsels patients with obesity and cardiovascular disease regarding weight and exercise (range 0-5) & $4.8(.45)$ \\
\hline How often counsels patients with obesity and without cardiovascular disease regarding weight and exercise (range (0-5) & $5.0(0)$ \\
\hline Approximate time per visit spent counseling patients regarding weight and exercise & $11.67(3.41)$ \\
\hline
\end{tabular}


Table 2

Example of Session Documentation Form

\begin{tabular}{|l|l|}
\hline Session: 123456789101112 & \\
\hline Name: & Date: \\
\hline Weight: & Physical Activity (min/week): \\
\hline General Assessment (any life change or issue since last visit, any questions or concerns): \\
\hline HEALTHY EATING & Comments \\
\hline \multicolumn{2}{|l|}{} \\
\hline Assess diet & \\
\hline Meeting goals set at last session? & \\
\hline If yes, identify successful strategies and skip to goals for next session. & \\
\hline If no, how ready and confident to change? & \\
\hline If no, what are barriers? & \\
\hline Problem-solving (what could you do differently?) & \\
\hline Goals for next session & \\
\hline PHYSICAL ACTIVITY & \\
\hline & Comments \\
\hline Assess physical activity & \\
\hline Meeting goals set at last session? & \\
\hline If yes, identify successful strategies and skip to goals for next session. & \\
\hline If no, how ready and confident to change? & \\
\hline If no, what are barriers? & \\
\hline Problem-solving (what could you do differently?) & \\
\hline Goals for next session & \\
\hline
\end{tabular}


Table 3

Example of Components of Lifestyle Program

\begin{tabular}{|l|l|}
\hline Behavioral Strategies & $\begin{array}{l}\text { Goal setting } \\
\text { Self-monitoring (documenting food intake and physical activity) } \\
\text { Problem-solving barriers } \\
\text { Assessing readiness and confidence to change } \\
\text { Motivational interviewing }\end{array}$ \\
\hline Education & Benefits of 5-7\% weight loss in preventing diabetes \\
& Healthy food choices (choosing meals and snacks from variety of foods, decreasing saturated fat, high fiber \\
& carbohydrates) \\
& Portion control \\
& Understanding food labels \\
& Menus and recipe modification \\
& Meal replacement \\
& Emotional eating \\
& Healthy eating out \\
& Benefits of exercise and exercise safety \\
& Pedometers \\
& Tips on being active \\
& Relapse prevention \\
& Stimulus control (eg., eliminating junk food from home environment) \\
& Stress management \\
& Ways to stay motivated \\
\hline
\end{tabular}

